\title{
ELLIPTIC CURVES WITH TORSION GROUP $\mathbb{Z} / 6 \mathbb{Z}$
}

\author{
Andrej Dujella, Juan Carlos Peral and Petra Tadić \\ University of Zagreb, Croatia, Universidad del País Vasco, Spain and Juraj \\ Dobrila University of Pula, Croatia
}

\begin{abstract}
We exhibit several families of elliptic curves with torsion group isomorphic to $\mathbb{Z} / 6 \mathbb{Z}$ and generic rank at least 3 . Families of this kind have been constructed previously by several authors: Lecacheux, Kihara, Eroshkin and Woo. We mention the details of some of them and we add other examples developed more recently by Dujella and Peral, and MacLeod.

Then we apply an algorithm of Gusić and Tadić and we find the exact rank over $\mathbb{Q}(t)$ to be 3 and we also determine free generators of the Mordell-Weil group for each family. By suitable specializations, we obtain the known and new examples of curves over $\mathbb{Q}$ with torsion $\mathbb{Z} / 6 \mathbb{Z}$ and rank 8 , which is the current record.
\end{abstract}

\section{INTRODUCTION}

Families of curves with torsion group $\mathbb{Z} / 6 \mathbb{Z}$ and rank at least 3 have been constructed previously by several authors, Lecacheux ([11]), Kihara ([9]) and later on Eroshkin ([6]) and Woo ([16]) are among them. More recently, Dujella and Peral (unpublished, 2012) and MacLeod ([12]) found other examples.

We detail some of these families, and in each case we include the relevant data and the output of the application of the algorithm of Gusic and Tadic $([8])$, to determine the exact rank over $\mathbb{Q}(t)$ and free generators of the MordellWeil group. We apply [8, Theorem 1.3], which deals with elliptic curves $E$ given by $y^{2}=x^{3}+A(t) x^{2}+B(t) x$, where $A, B \in \mathbb{Z}[t]$, with exactly one nontrivial 2-torsion point over $\mathbb{Q}(t)$. If $t_{0} \in \mathbb{Q}$ satisfies the condition that for every nonconstant square-free divisor $h$ of $B(t)$ or $A(t)^{2}-4 B(t)$ in $\mathbb{Z}[t]$

2010 Mathematics Subject Classification. 11G05.

Key words and phrases. Elliptic curves, torsion, rank.

A. D. and P. T. were supported by the Croatian Science Foundation under the project no. 6422. J. C. P. was supported by the UPV/EHU grant EHU 10/05. A. D. acknowledges support from the QuantiXLie Center of Excellence. 
the rational number $h\left(t_{0}\right)$ is not a square in $\mathbb{Q}$, then the specialized curve $E_{t_{0}}$ is elliptic and the specialization homomorphism at $t_{0}$ is injective. If additionally there exist $P_{1}, \ldots, P_{r} \in E(\mathbb{Q}(t))$ such that $P_{1}\left(t_{0}\right), \ldots, P_{r}\left(t_{0}\right)$ are free generators of $E\left(t_{0}\right)(\mathbb{Q})$, then $E(\mathbb{Q}(t))$ and $E\left(t_{0}\right)(\mathbb{Q})$ have the same rank $r$, and $P_{1}, \ldots, P_{r}$ are free generators of $E(\mathbb{Q}(t))$.

Let us mention that the methods from [8] have been used in [3] to determine the exact rank over $\mathbb{Q}(t)$ of some of record rank families with torsion groups $\mathbb{Z} / 4 \mathbb{Z}, \mathbb{Z} / 10 \mathbb{Z}, \mathbb{Z} / 12 \mathbb{Z}$ and $\mathbb{Z} / 2 \mathbb{Z} \times \mathbb{Z} / 8 \mathbb{Z}$, while the analogous result for torsion group $\mathbb{Z} / 2 \mathbb{Z} \times \mathbb{Z} / 4 \mathbb{Z}$ has been obtained in $[4]$ by using previous version of the injectivity criterium by Gusić and Tadic from [7].

\section{Curves with torsion group $\mathbb{Z} / 6 \mathbb{Z}$}

The Tate's normal form for an elliptic curve is given by

$$
E(b, c): \quad y^{2}+(1-c) x y-b y=x^{3}-b x^{2},
$$

see $[10$, p. 147]. It is nonsingular if and only if $b \neq 0$. Using the addition law for the point $P=(0,0)$ we find

$$
\begin{aligned}
3 P & =(c, b-c), \\
-3 P & =\left(c, c^{2}\right) .
\end{aligned}
$$

It follows that $P$ is a torsion point of order 6 for $b=c+c^{2}$. So, for $b=c+c^{2}$ we write the curve in the form $y^{2}=x^{3}+A(c) x^{2}+B(c) x$. We have

$$
\begin{aligned}
& A(c)=1+6 c-3 c^{2} \\
& B(c)=-16 c^{3}
\end{aligned}
$$

In the new coordinates the torsion point of order 6 is $(-4 c, 4 c(1+c))$.

\section{LECACHEUX' CONSTRUCTION FOR RANK 3}

3.1. Deduction of the coefficients for a rank 3 family. A rank 3 family comes from specialization in the rank 2 family given by Lecacheux ([11]).

In this paper Lecacheux constructs four biparametric families of curves with torsion group $\mathbb{Z} / 6 \mathbb{Z}$ and rank 2 over $\mathbb{Q}(s, t)$, and by specialization of $s$ she gets a family of curves with this torsion and rank 3 over $\mathbb{Q}(t)$. The starting model used by Lecacheux for torsion $\mathbb{Z} / 6 \mathbb{Z}$, once translated to $(0,0)$, is $y^{2}=x^{3}+a_{6}(d) x^{2}+b_{6}(d) x$ where

$$
\begin{aligned}
& a_{6}(d)=-3-6 d+d^{2}, \\
& b_{6}(d)=16 d .
\end{aligned}
$$


Lecacheux shows that using any of the four values

$$
\begin{aligned}
& d_{1}=\frac{\left(-t^{2}+s\right)(1+t s)\left(t-s^{2}\right)}{(-1+t) t(-1+s) s(t+s)} \\
& d_{2}=\frac{\left(-s^{2}+t(1+t)(1+s)\right)\left(-t^{2}+(1+t) s(1+s)\right)}{t s(1+t+s)(t+s+t s)} \\
& d_{3}=\frac{(t+s+t s(2+t+s))\left((1+t+s)^{2}+t s(1+t s+2(t+s))\right)}{t\left(1+t+t^{2}\right) s\left(1+s+s^{2}\right)} \\
& d_{4}=-\frac{(t-1)(s-1)\left(t^{2}+t s+s^{2}\right)(1+t s+2(t+s))}{(t+s)\left(-t^{2}+s\right)\left(t-s^{2}\right)}
\end{aligned}
$$

the resulting biparametric family has rank $\geq 2$ over $\mathbb{Q}(s, t)$. Then she specializes and gets a family of rank 3 over $\mathbb{Q}(t)$. The value of $d$ is a rational expression with numerator of degree 10 and denominator of degree 9 given by:

$$
\frac{\left(-1+t+t^{2}\right)\left(-1+2 t+t^{3}\right)\left(-1+2 t-t^{2}+t^{3}+2 t^{4}+t^{5}\right)}{t^{2}(1+t)(-1+2 t)\left(-1+2 t+t^{2}\right)\left(-1+t+t^{2}+t^{3}\right)} .
$$

The curve of rank 3 has polynomial coefficients in $t$ of degree 20 and 40 respectively. The coefficients are

$$
\begin{aligned}
a_{63 L}(t)= & 1-10 t+35 t^{2}-36 t^{3}-61 t^{4}+146 t^{5}+5 t^{6}-254 t^{7}+300 t^{8}+58 t^{9} \\
& -436 t^{10}-294 t^{11}+496 t^{12}+710 t^{13}+93 t^{14}-434 t^{15}-489 t^{16} \\
& -264 t^{17}-73 t^{18}-6 t^{19}+t^{20}, \\
b_{63 L}(t)= & 16 t^{6}(1+t)^{3}(-1+2 t)^{3}\left(-1+t+t^{2}\right)\left(-1+2 t+t^{2}\right)^{3}\left(-1+2 t+t^{3}\right) \\
& \times\left(-1+t+t^{2}+t^{3}\right)^{3}\left(-1+2 t-t^{2}+t^{3}+2 t^{4}+t^{5}\right) .
\end{aligned}
$$

and the $x$-coordinates of three infinite order independent points $P_{1}, P_{2}$ and $P_{3}$ are

$$
\begin{gathered}
x\left(P_{1}\right)=4 t(1+t)(-1+2 t)\left(-1+t+t^{2}\right)\left(-1+2 t+t^{2}\right)^{2}\left(-1+2 t+t^{3}\right) \\
\left(-1+t+t^{2}+t^{3}\right)^{2}, \\
x\left(P_{2}\right)=4 t^{4}(1+t)(-1+2 t)\left(-1+2 t+t^{2}\right)^{2}\left(-1+2 t+t^{3}\right) \\
\left(-1+2 t-t^{2}+t^{3}+2 t^{4}+t^{5}\right), \\
x\left(P_{3}\right)=4 t^{2}(-1+2 t)\left(-1+2 t+t^{2}\right)^{2}\left(-1+t+t^{2}+t^{3}\right)^{2} \\
\left(-1+2 t-t^{2}+t^{3}+2 t^{4}+t^{5}\right) .
\end{gathered}
$$

3.2. Generators. Now we prove that the elliptic curve $C$ over $\mathbb{Q}(t)$ given by the equation

$C: y^{2}=x^{3}+a_{63 L} x^{2}+b_{63 L} x, \quad\left(\right.$ where $a_{63 L}, b_{63 L}$ are given above) 
has rank equal 3 and the points $P_{1}, P_{2}, P_{3}$ are its free generators. We apply methods from [8], as explained in the introduction.

- We use the specialization at $t_{0}=-7$ which is injective by $[8$, Theorem $1.3]$.

- The specialized curve $C^{-7}$ over $\mathbb{Q}$ with reduced coefficients is $[0,298972834764046,0,-4129733728640949525768711375,0]$.

- By mwrank $([1])$, the rank of this specialized curve over $\mathbb{Q}$ is equal to 3 and its free generators are

$$
\begin{aligned}
& G_{1}=[-242529733107900,-2078846881485761806650], \\
& G_{2}=[-39216968008071,-749301352264289045760], \\
& G_{3}=[31096987486425,436763906196136192800] .
\end{aligned}
$$

- We have that for the specialization of the points $P_{1}, P_{2}, P_{3}$ at $t_{0}=-7$ it holds

$$
\begin{aligned}
& P_{1}^{-7}=3 \cdot T+0 \cdot G_{1}-1 \cdot G_{2}-1 \cdot G_{3}, \\
& P_{2}^{-7}=1 \cdot T+1 \cdot G_{1}+0 \cdot G_{2}-1 \cdot G_{3}, \\
& P_{3}^{-7}=5 \cdot T+0 \cdot G_{1}-1 \cdot G_{2}+0 \cdot G_{3},
\end{aligned}
$$

where $T$ is the torsion point of order 6 on the specialized curve.

- Since the determinant of the base change matrix has absolute value 1 , it follows that the points $P_{1}, P_{2}, P_{3}$ with $x$-coordinates given above are free generators of $C$ over $\mathbb{Q}(t)$.

\section{KiHARA's CONSTRUCTION FOR RANK 3}

4.1. Family data. Kihara ([9]) considers the projective curve

$$
\left(x^{2}-y^{2}\right)^{2}+\left(2 a x^{2}+2 b y^{2}\right) z^{2}+c z^{4}=0,
$$

then he uses the substitutions

$$
\begin{aligned}
& X=\frac{x^{2}}{y^{2}}, \\
& Y=\frac{x\left(c z^{2}+a x^{2}+b y^{2}\right)}{y^{3}},
\end{aligned}
$$

and arrives to the elliptic curve

$$
E: \quad Y^{2}=X\left(\left(a^{2}-c\right) X^{2}+(2 a b+2 c) X+\left(b^{2}-c\right)\right) .
$$

The point $P=(1, a+b)$ is on $E$. Forcing $P$ to be of order 3 implies $c=$ $\frac{(3 a-b)(a+b)}{4}$ in which case $P+(0,0)$ is of order 6 . With this value of $c$ the curve $E$ is given by $Y^{2}=X^{3}+A(a, b) X^{2}+B(a, b) X$ where

$$
\begin{aligned}
& A(a, b)=2\left(3 a^{2}+6 a b-b^{2}\right), \\
& B(a, b)=-(a-b)^{3}(3 a+5 b) .
\end{aligned}
$$


By imposing new points on the curve, Kihara gets a family with torsion group $\mathbb{Z} / 6 \mathbb{Z}$ and rank at least 3 given as $y^{2}=x^{3}+a_{63 K}(t) x^{2}+b_{63 K}(t) x$ where

$$
\begin{aligned}
a_{63 K}(t)= & -2\left(64 t^{8}-1952 t^{7}-4652 t^{6}-10172 t^{5}-28955 t^{4}+35602 t^{3}\right. \\
& \left.-56987 t^{2}+83692 t+9604\right), \\
b_{63 K}(t)= & (t-7)^{3}(t+2)^{3}(2 t+1)^{3}(4 t-7)^{3}\left(2 t^{2}-91 t+98\right)\left(4 t^{2}+13 t+1\right) .
\end{aligned}
$$

The $x$-coordinates of three independent points of infinite order are:

$$
\begin{aligned}
& x\left(Q_{1}\right)=\frac{9(-7+t)^{2}(2+t)^{2}(1+2 t)^{2}(-7+4 t)^{2}\left(7+2 t^{2}\right)^{2}}{\left(-7-4 t+2 t^{2}\right)^{2}}, \\
& x\left(Q_{2}\right)=\frac{(-7+t)^{2}(2+t)^{2}(1+2 t)^{2}(-7+4 t)^{2}\left(7+2 t^{2}\right)^{2}}{\left(-7+14 t+2 t^{2}\right)^{2}}, \\
& x\left(Q_{3}\right)=\frac{(-7+t)^{2}(2+t)^{2}(1+2 t)^{2}(-7+4 t)^{2}\left(-7+14 t+2 t^{2}\right)^{2}}{\left(-7-4 t+2 t^{2}\right)^{2}} .
\end{aligned}
$$

4.2. Generators. Now following the modified proof of subsection 3.2 we prove that Kihara's elliptic curve over $\mathbb{Q}(t)$ given at the end of subsection 4.1 has rank equal to 3 and the points $W_{1}, W_{2}, Q_{3}$ are its free generators (the $x$-coordinate of $Q_{3}$ given above, and of $W_{1}, W_{2}$ given below). So the above given points $Q_{1}, Q_{2}, Q_{3}$ found by Kihara, are not its free generators.

- The specialization at $t_{0}=15$ is injective by [8, Theorem 1.3].

- The reduced coefficients of the specialized curve are $[0,7236353038,0,-2438945400771712139,0]$.

- By mwrank ([1]), the specialized curve has rank equal to 3 and its free generators are

$$
\begin{aligned}
& G_{1}=[-3875031,-3091858818006], \\
& G_{2}=[5184423886489 / 64,-12318461250340505085 / 512], \\
& G_{3}=[83648786841 / 4,-28010191585888407 / 8] .
\end{aligned}
$$

- We have that the specialization of the points $Q_{1}, Q_{2}, Q_{3}$ at $t_{0}=15$ satisfies

$$
\begin{aligned}
& Q_{1}^{15}=0 \cdot T+2 \cdot G_{1}+0 \cdot G_{2}+1 \cdot G_{3}, \\
& Q_{2}^{15}=0 \cdot T+2 \cdot G_{1}+2 \cdot G_{2}+3 \cdot G_{3}, \\
& Q_{3}^{15}=4 \cdot T+0 \cdot G_{1}+0 \cdot G_{2}-1 \cdot G_{3},
\end{aligned}
$$

here $T$ is the torsion point of order 6 on the specialized curve.

- From this we see that we can take points $W_{1}$ and $W_{2}$ such that

$$
\begin{aligned}
Q_{1}+Q_{3} & =2 W_{1}, \\
Q_{2}+3 Q_{3} & =2 W_{2} .
\end{aligned}
$$


- The $x$-coordinates of $W_{1}$ and $W_{2}$ are

$$
\begin{aligned}
x\left(W_{1}\right)= & (4 t-7)(t-7)\left(4 t^{2}+13 t+1\right)\left(2 t^{2}-7 t+14\right)^{2}, \\
x\left(W_{1}\right)= & (t+2)^{2}\left(4 t^{2}+13 t+1\right)\left(64 t^{5}-536 t^{4}+1324 t^{3}+224 t^{2}-490 t-343\right)^{2} \\
& \times(2 t+1)^{2}(t-7)(4 t-7) /\left(64 t^{5}-8 t^{4}-284 t^{3}+44 t^{2}+476 t-49\right)^{2} .
\end{aligned}
$$

- Now it is obvious that $W_{1}, W_{2}, Q_{3}$ are free generators of the observed elliptic curve over $\mathbb{Q}(t)$. More precisely

$$
\begin{aligned}
W_{1}^{15} & =1 \cdot T-1 \cdot G_{1}+0 \cdot G_{2}+0 \cdot G_{3}, \\
W_{2}^{15} & =3 \cdot T+1 \cdot G_{1}+1 \cdot G_{2}+3 \cdot G_{3}, \\
Q_{3}^{15} & =4 \cdot T+0 \cdot G_{1}+0 \cdot G_{2}-1 \cdot G_{3} .
\end{aligned}
$$

\section{EROSHKIN'S CONSTRUCTION FOR RANK 3}

5.1. Family data. In 2008, Eroshkin ([6]) constructed another example of rank 3 curve over $\mathbb{Q}(t)$. The starting point for his construction is the twoparametric family with rank $\geq 2$ over $\mathbb{Q}(u, v)$, given by

$$
\begin{aligned}
A(u, v)= & v^{8} u^{4}+2 u^{7} v^{5}+3 u^{6} v^{6}+2 u^{5} v^{7}+3 u^{4} v^{2}+3 v^{4} u^{2}-12 u^{3} v^{3}+u^{8} v^{4} \\
& -3 u^{6}-6 u^{5}+7 u^{4}-3 u^{2}+6 u^{3}-6 v^{5}+7 v^{4}-3 v^{6}+21 u^{6} v^{2} \\
& +36 u^{4} v^{4}-9 u^{2} v^{2}+12 v^{2} u-24 u^{4} v^{5}-9 u^{4} v^{6}+36 u^{5} v^{3}+21 u^{2} v^{6} \\
& -24 u^{5} v^{4}-10 v^{4} u-6 v^{7} u^{2}+4 u^{5} v^{6}-2 u^{2} v^{5}+2 u^{4} v^{7}-14 u^{3} v^{6} \\
& +8 u^{5} v-9 u^{6} v^{4}-18 u^{5} v^{5}+2 v^{7} u^{3}+12 v u^{2}+2 u^{3} v-6 v^{6} u \\
& -20 u^{2} v^{3}+8 v^{5} u-6 u^{6} v+36 u^{3} v^{5}+2 u^{7} v^{3}-10 u^{4} v+4 u^{6} v^{5}-6 u v \\
& +2 v^{3} u-6 u^{7} v^{2}-14 u^{6} v^{3}-3 v^{2}+6 v^{3}+2 u^{7} v^{4}-2 u^{5} v^{2}-20 u^{3} v^{2}, \\
B(u, v)= & -16(u+v)^{3}(-1+v)^{3}(1+v)^{3}(-1+u)^{3}(1+u)^{3} \\
& \times\left(u^{2}+u v+v^{2}-2 v u^{2}-2 v^{2} u+u^{3} v+v^{3} u-2 u^{3} v^{2}-2 u^{2} v^{3}\right. \\
& \left.+u^{4} v^{2}+v^{4} u^{2}+u^{3} v^{3}\right) .
\end{aligned}
$$

The curve $y^{2}=x^{3}+A(u, v) x^{2}+B(u, v) x$ contains the points with $x$ coordinates $4(v-1)^{2}(1+v)^{3}(-1+u)^{2}(1+u)^{3}$ and $-4(u-1)(u+1)(v-$ $1)^{3}(v+1)^{2}(u+v)^{3}$. Forcing the point with $x$-coordinate $3(v-1)^{2}(v+1)^{2}(-1+$ $u)^{2}(1+u)^{2}(v+u)^{2}$ to belong to the curve, leads to the condition $v=1 / 3$. By substitution $u=(1-t) /(1+t)$ and some simplifications, we get Eroshkin's rank 3 curve with coefficients

$$
\begin{aligned}
& a_{63 E}(t)=16+576 t-1408 t^{2}-1440 t^{3}+1608 t^{4}+720 t^{5}-352 t^{6}-72 t^{7}+t^{8}, \\
& b_{63 E}(t)=27648(-2+t)^{3} t^{3}(1+t)^{3}\left(2+t^{2}\right)^{2},
\end{aligned}
$$


and $x$-coordinates of three independent points $R_{1}, R_{2}, R_{3}$ given by:

$$
\begin{aligned}
& x\left(R_{1}\right)=864(-2+t)^{3} t^{3}, \\
& x\left(R_{2}\right)=3456 t^{2}(1+t)^{3}, \\
& x\left(R_{3}\right)=288(-2+t)^{3} t^{3}(1+t)^{2} .
\end{aligned}
$$

Another way to get a rank 3 family from Eroshkin's two-parametric family is to take $v=-1 / 3$. By substitution $u=(1-t) /(1+t)$, as above, we get

$$
\begin{aligned}
a_{63 E R}(t)= & 256 t^{8}-2304 t^{7}-3232 t^{6}+1008 t^{5}+2337 t^{4}-504 t^{3}-808 t^{2} \\
& +288 t+16, \\
b_{63 E R}(t)= & 27648\left(16 t^{4}-11 t^{2}+4\right)(2 t-1)^{3}(t+1)^{3} t^{3},
\end{aligned}
$$

and three infinite order independent points $S_{1}, S_{2}, S_{3}$ with $x$-coordinates

$$
\begin{aligned}
& x\left(S_{1}\right)=1728 t^{2}(t+1)^{3}, \\
& x\left(S_{2}\right)=864 t^{3}(2 t-1)^{3}, \\
& x\left(S_{3}\right)=864 t^{3}(t+1)^{2}(2 t-1) .
\end{aligned}
$$

5.2. Generators. We prove that the rank of both families (for $v=1 / 3$ and $v=-1 / 3$ ) is equal to 3 and the points $R_{1}, R_{2}, R_{3}$ (resp. $S_{1}, S_{2}, S_{3}$ ) are free generators of the elliptic curve $y^{2}=x^{3}+a_{63 E} x^{2}+b_{63 E} x$ (resp. $\left.y^{2}=x^{3}+a_{63 E R} x^{2}+b_{63 E R} x\right)$ over $\mathbb{Q}(t)$, where $a_{63 E}, b_{63 E}, a_{63 E R}, b_{63 E R}$ are given above.

- For the first curve, we use the specialization at $t_{0}=-11$ which is injective by [8, Theorem 1.3].

- The specialized curve over $\mathbb{Q}$ with reduced coefficients is

$$
[0,100352409,0,-15100698522624000,0] \text {. }
$$

- By mwrank ([1]), the rank of this specialized curve over $\mathbb{Q}$ is equal to 3 and its free generators are

$$
\begin{aligned}
G_{1} & =[-11073920,-422104608640], \\
G_{2} & =[-21632000,-602905472000], \\
G_{3} & =[879994086400 / 9409,-486030413853670400 / 912673] ;
\end{aligned}
$$

- For the specialization of the points $R_{1}, R_{2}, R_{3}$ at $t_{0}=-11$ we have

$$
\begin{aligned}
& R_{1}^{-11}=5 \cdot T+0 \cdot G_{1}+1 \cdot G_{2}+1 \cdot G_{3}, \\
& R_{2}^{-11}=5 \cdot T+1 \cdot G_{1}-1 \cdot G_{2}-1 \cdot G_{3}, \\
& R_{3}^{-11}=0 \cdot T-1 \cdot G_{1}+1 \cdot G_{2}+0 \cdot G_{3},
\end{aligned}
$$

where $T$ is the torsion point of order 6 on the specialized curve.

- Since the determinant of the base change matrix has absolute value 1 , it follows that the points $R_{1}, R_{2}, R_{3}$ with $x$-coordinates given above are free generators of $y^{2}=x^{3}+a_{63 E} x^{2}+b_{63 E} x$ over $\mathbb{Q}(t)$. 
- For the second curve we use the specialization at $t_{0}=-15$ which is injective by [8, Theorem 1.3].

- The specialized curve over $\mathbb{Q}$ is

$$
[0,1012299875521,0,-6159774508321416192000,0] .
$$

- By mwrank $([1])$, the rank of this specialized curve over $\mathbb{Q}$ is equal to 3 and its free generators are

$$
\begin{aligned}
& G_{1}=[4710284355840,11266618646136456960], \\
& G_{2}=[-70907506432,-71902551469760256], \\
& G_{3}=\left[\frac{21570079809600}{169},-\frac{292982182574714539200}{2197}\right] .
\end{aligned}
$$

- We have that for the specialization of the points $S_{1}, S_{2}, S_{3}$ at $t_{0}=-15$ it holds

$$
\begin{aligned}
& S_{1}^{-15}=1 \cdot T-1 \cdot G_{1}+0 \cdot G_{2}-1 \cdot G_{3}, \\
& S_{2}^{-15}=3 \cdot T+0 \cdot G_{1}+1 \cdot G_{2}+0 \cdot G_{3}, \\
& S_{3}^{-15}=1 \cdot T+0 \cdot G_{1}+1 \cdot G_{2}-1 \cdot G_{3},
\end{aligned}
$$

where $T$ is the torsion point of order 6 on the specialized curve.

- Since the determinant of the base change matrix has absolute value 1 , it follows that the points $S_{1}, S_{2}, S_{3}$ with $x$-coordinates given above are free generators of $y^{2}=x^{3}+a_{63 E R} x^{2}+b_{63 E R} x$ over $\mathbb{Q}(t)$.

\section{A direct construction by Dujella and Peral (2012)}

6.1. Deduction of the family. Our next example is based on the use of 2 -descent as follows, see e.g. [5]. Consider the general curve with torsion $\mathbb{Z} / 6 \mathbb{Z}$ as given in Section 2 i.e.:

$$
y^{2}=x^{3}+x^{2}\left(1+6 c-3 c^{2}\right)+x\left(-16 c^{3}\right) .
$$

We first impose $16 c^{2}$ as $x$-coordinate of a new point, this is equivalent to solve $1+5 c+13 c^{2}=\square$. The corresponding parametrization is $c=-\frac{(-4+u)(-2+u)}{-13+u^{2}}$. This gives us an infinite order point and a curve with rank at least 1 over $\mathbb{Q}(u)$ whose coefficients are

$$
\begin{aligned}
& A_{61}(u)=601-180 u-152 u^{2}+72 u^{3}-8 u^{4}, \\
& B_{61}(u)=16(-4+u)^{3}(-2+u)^{3}\left(-13+u^{2}\right) .
\end{aligned}
$$

Now observe that imposing $4(-4+u)(-2+u)^{3}$ as a new point is the same as to solve $-103+12 u+4 u^{2}=\square$. This can be achieved with $u=-\frac{103+t^{2}}{4(-3+t)}$. 
The new family has the following coefficients

$$
\begin{aligned}
A_{63}(t)= & -2\left(26009437+18059772 t+5057576 t^{2}+813612 t^{3}+89370 t^{4}\right. \\
& \left.+7860 t^{5}+608 t^{6}+36 t^{7}+t^{8}\right), \\
B_{63}(t)= & (5+t)^{3}(11+t)^{3}\left(79+8 t+t^{2}\right)^{3}\left(8737+1248 t-2 t^{2}+t^{4}\right) .
\end{aligned}
$$

In this case the family has rank $\geq 3$. Below we list the $x$-coordinates of three independent points of infinite order on the curve

$$
\begin{aligned}
& x\left(P_{1}\right)=4(5+t)^{2}(11+t)^{2}\left(79+8 t+t^{2}\right)^{2}, \\
& x\left(P_{2}\right)=(5+t)(11+t)\left(79+8 t+t^{2}\right)^{3}, \\
& x\left(P_{3}\right)=\frac{64(4+t)^{2}(5+t)^{2}(11+t)^{2}\left(79+8 t+t^{2}\right)^{2}}{\left(29+6 t+t^{2}\right)^{2}} .
\end{aligned}
$$

The first two points are the ones we have imposed in our constructions, while the third one appears in the last change. So this change produces two new independent points of infinite order and accordingly this family has rank at least 3 .

6.2. Generators. We prove the curve $y^{2}=x^{3}+A_{63} x^{2}+B_{63} x$ has rank exactly 3 over $\mathbb{Q}(t)$ with free generators the points $P_{1}, P_{2}, P_{3}$.

Here is the proof, it is similar to the proof in subsection 3.2.

- We take the specialization homomorphism at $t_{0}=13$ which is injective by $[8$, Theorem 1.3$]$.

- Reduced form of the specialized curve is

$$
[0,-3121367,0,2201786966016,0] \text {. }
$$

- Mwrank ([1]) shows that the rank of the specialized curve is equal to 3, and its free generators are

$$
\begin{aligned}
G_{1} & =[38016,281508480] \\
G_{2} & =[213276816,-3091894330320], \\
G_{3} & =[381655296 / 361,985650589440 / 6859] .
\end{aligned}
$$

We have that for the specialization of the points $P_{1}, P_{2}, P_{3}$ at $t_{0}=13$

$$
\begin{aligned}
& P_{1}^{-11}=0 \cdot T-2 \cdot G_{1}+1 \cdot G_{2}+0 \cdot G_{3}, \\
& P_{2}^{-11}=0 \cdot T-1 \cdot G_{1}+0 \cdot G_{2}-1 \cdot G_{3}, \\
& P_{3}^{-11}=2 \cdot T+2 \cdot G_{1}-1 \cdot G_{2}+1 \cdot G_{3},
\end{aligned}
$$

where $T$ is the torsion point of order 6 on the specialized curve.

- The points with $x$-coordinates $P_{1}(t), P_{2}(t), P_{3}(t)$ are free generators of the full Mordell-Weil group, analogously as before. 


\section{The construction by MacLeod (2013)}

7.1. Coefficients for a rank 3 family. A rank 3 family comes from the paper of MacLeod [12]. The curve of rank 3 has polynomial coefficients:

$$
\begin{aligned}
a_{M L}(t)= & 60637 t^{16}-9446576 t^{15}+318481560 t^{14}+7559095920 t^{13} \\
& -673080292884 t^{12}+13070347480656 t^{11}-22695122540632 t^{10} \\
& -1545816592882960 t^{9}-5109785850000978 t^{8} \\
& +496074059556450416 t^{7}-4896348487968033496 t^{6} \\
& +8785946885865627600 t^{5}+127778653981127482476 t^{4} \\
- & 861560195308301691408 t^{3}+2072099226498542082072 t^{2} \\
& -1499601599678467324208 t-1357666940926062868067, \\
b_{M L}(t)= & -\left(t^{2}-14 t-83\right)^{3}\left(17 t^{2}-226 t-367\right)^{3}\left(t^{2}+58 t-347\right)^{3} \\
\times & \left(t^{2}-290 t+2017\right)^{3} \\
\times & \left(445 t^{8}-26680 t^{7}+298540 t^{6}+7487800 t^{5}-100559026 t^{4}\right. \\
& \left.-816359048 t^{3}+14922867436 t^{2}-55316709112 t+68821828189\right),
\end{aligned}
$$

and the $x$-coordinates of three infinite order independent points $Q_{1}, Q_{2}$ and $Q_{3}$ are

$$
\begin{aligned}
x\left(Q_{1}\right)= & -4\left(13 t^{2}-458 t+1021\right)^{2}\left(t^{2}+4 t-149\right)^{2}\left(t^{2}-290 t+2017\right) \\
& \left(t^{2}+58 t-347\right)\left(17 t^{2}-226 t-367\right)\left(t^{2}-14 t-83\right), \\
x\left(Q_{2}\right)= & -9\left(17 t^{2}-226 t-367\right)^{3}\left(t^{2}-14 t-83\right)^{3}\left(t^{2}-290 t+2017\right) \\
& \times\left(t^{2}+58 t-347\right), \\
x\left(Q_{3}\right)= & \left(445 t^{8}-26680 t^{7}+298540 t^{6}+7487800 t^{5}-100559026 t^{4}\right. \\
& \left.-816359048 t^{3}+14922867436 t^{2}-55316709112 t+68821828189\right) \\
& \times\left(17 t^{2}-226 t-367\right)^{2}\left(t^{2}-14 t-83\right)^{2} .
\end{aligned}
$$

7.2. Generators. Following the proof in subsection 3.2 we prove that MacLeod's elliptic curve over $\mathbb{Q}(t)$ given in subsection 7.1 has rank equal to 3 over $\mathbb{Q}(t)$ and the points $Q_{1}, Q_{2}, Q_{3}$ are its free generators.

- We use the specialization at $t_{0}=-30$ which is injective by $[8$, Theorem 1.3].

- The specialized curve over $\mathbb{Q}$ already has the reduced coefficients

$$
[0,-396608000022758283110679701027,0,
$$

$44041764841344163001873681355897427474904948473305010381651,0]$. 
- By mwrank $([1])$, the rank of this specialized curve over $\mathbb{Q}$ is equal to 3 and its free generators are

$G_{1}=[1371227854300350505685906632351$,

$1375839998833531665451365906419334737742953475]$,

$G_{2}=\left[\frac{11719103414610661317429257961549638649}{259596544}\right.$,

- $\left.\frac{149169932197111418433971723731890785699819555746182187635}{4182619516928}\right]$

$G_{3}=\left[\frac{3986901670582496234640210004431381477056020081}{33036918984050881}\right.$,

$\left.-\frac{216209573005548181556962517949975521622133551319748523058488502950375}{6004810514024749417546529}\right]$.

- We have that for the specialization of the points $Q_{1}, Q_{2}, Q_{3}$ at $t_{0}=-30$ it holds

$$
\begin{aligned}
& Q_{1}^{-30}=0 \cdot T+1 \cdot G_{1}+1 \cdot G_{2}-1 \cdot G_{3}, \\
& Q_{2}^{-30}=2 \cdot T+1 \cdot G_{1}+0 \cdot G_{2}-1 \cdot G_{3}, \\
& Q_{3}^{-30}=1 \cdot T-1 \cdot G_{1}+0 \cdot G_{2}+0 \cdot G_{3},
\end{aligned}
$$

where $T$ is the torsion point of order 6 on the specialized curve.

- Since the determinant of the base change matrix has absolute value 1 , it follows that the points $Q_{1}, Q_{2}, Q_{3}$ with $x$-coordinates given above are free generators of the observed curve over $\mathbb{Q}(t)$.

\section{Examples With RANK 8 OVER $\mathbb{Q}$}

Currently the highest know rank for curves over $\mathbb{Q}$ with torsion group $\mathbb{Z} / 6 \mathbb{Z}$ is 8 . In this section, we explain how 10 previously known and 10 new curves with rank 8 were (re)discovered. The details (minimal equation, torsion points, independent points of infinite order) for all these curve can be found at $\mathbb{Z} / 6 \mathbb{Z}$ section of tables in $[2]$.

We applied standard techniques for finding curves with high rank in families with reasonably large generic rank. These techniques include computations of Mestre-Nagao sums $([13,15])$ and 2-Selmer rank. In our computations we use PARI/GP ([14]) and mwrank ([1]). In most of the families mentioned in previous sections, we are able to find curves with rank at least 7 . Here we report only constructions which give curves with rank 8 .

8.1. Kihara's family. By applying the above mentioned techniques to Kihara's family of rank 3 over $\mathbb{Q}(t)$ from Section 4 , we find the curves with rank 8 over $\mathbb{Q}$ for the following values of the parameter $t$ :

- 39/121 (2-isogenous to 4th curve in [2]; Elkies (2008)),

- 178/113 (2-isogenous to 5th curve in [2]; Elkies (2008)),

- 527/301 (8th curve in [2]; Dujella (2008)),

- $-1 / 839$ (9th curve in [2]; Dujella (2008)),

- 519/407 (7th curve in [2]; Dujella (2008)), 
- $722 / 211$ (6th curve in [2]; Elkies (2008)),

- $-601 / 383$ (11th curve in [2]; Dujella-Peral-Tadić (2014)),

- -909/652 (12th curve in [2]; Dujella-Peral-Tadić (2014)).

8.2. Family by Dujella and Peral. In the family of rank 3 by Dujella and Peral from Section 6 we found only one curve with rank 8 , for $t=-244 / 3$. It is 2-isogenous to 10th curve in [2], listed there as Dujella-Peral (2012).

8.3. Eroshkin's two-parametric family. In Eroshkin's family of rank 3 over $\mathbb{Q}(t)$ obtained for $v=1 / 3$ we were able to find only examples with rank 7 . On the other hand, in the family of rank 3 obtained for $v=-1 / 3$ we have found four examples with rank 8 . Moreover, several examples with rank 8 were found within Eroshkin's two-parametric family with rank $\geq 2$ over $\mathbb{Q}(u, v)$. Here we list parameters $(u, v)$ corresponding to these examples:

- $(20 / 19,-7 / 17)$ (1st curve in [2]; Eroshkin (2008)),

- $(-4 / 25,7 / 19)$ (2nd curve in [2]; Eroshkin-Dujella (2008)),

- $(-73 / 80,11 / 7)$ (3rd curve in [2]; Eroshkin-Dujella (2008)),

- $(-1 / 31,21 / 13)$ (13th curve in [2]; Dujella-Peral-Tadić (2014)),

- $(101 / 119,-20 / 17)$ (14th curve in [2]; Dujella-Peral-Tadić (2014)),

- $(77 / 338,-1 / 3)$ (15th curve in [2]; Dujella-Peral-Tadić (2015)),

- $(633 / 229,-1 / 3)$ (16th curve in [2]; Dujella-Peral-Tadić (2015)),

- $(-283 / 437,6 / 19)$ (17th curve in [2]; Dujella-Peral-Tadić (2015)),

- $(-299 / 1395,-1 / 3)$ (18th curve in [2]; Dujella-Peral-Tadić (2015)),

- $(109 / 466,-7 / 8)$ (19th curve in [2]; Dujella-Peral-Tadić (2015)),

- $(447 / 305,-1 / 3)$ (20th curve in [2]; Dujella-Peral-Tadić (2015)).

\section{REFERENCES}

[1] J. Cremona, Algorithms for modular elliptic curves, Cambridge University Press, Cambridge, 1997.

[2] A. Dujella, High rank elliptic curves with prescribed torsion, http://web.math.hr/ $\sim$ duje/tors/tors.html.

[3] A. Dujella, I. Gusić and P. Tadić, The rank and generators of Kihara's elliptic curve with torsion $\mathbb{Z} / 4 \mathbb{Z}$ over $\mathbb{Q}(t)$, Proc. Japan Acad. Ser. A Math. Sci. 91 (2015), 105-109.

[4] A. Dujella and J. C. Peral, High rank elliptic curves with torsion $\mathbb{Z} / 2 \mathbb{Z} \times \mathbb{Z} / 4 \mathbb{Z}$ induced by Diophantine triples, LMS J. Comput. Math. 17 (2014), 282-288.

[5] A. Dujella, J.C. Peral, Elliptic curves with torsion group $\mathbb{Z} / 8 \mathbb{Z}$ or $\mathbb{Z} / 2 \mathbb{Z} \times \mathbb{Z} / 6 \mathbb{Z}$, in Trends in Number Theory, Contemp. Math. 649 (2015), 47-62.

[6] Y. G. Eroshkin, personal communication, 2008.

[7] I. Gusić and P. Tadić, A remark on the injectivity of the specialization homomorphism, Glas. Mat. Ser. III 47 (2012), 265-275.

[8] I. Gusić and P. Tadić, Injectivity of the specialization homomorphism of elliptic curves, J. Number Theory 148 (2015), 137-152.

[9] S. Kihara, On the rank of the elliptic curves with a rational point of order 6, Proc. Japan Acad. Ser. A Math. Sci. 82 (2006), 81-82.

[10] A. Knapp, Elliptic curves, Princeton University Press, 1992.

[11] O. Lecacheux, Rang de courbes elliptiques avec groupe de torsion non trivial, J. Théor. Nombres Bordeaux 15 (2003), 231-247. 
[12] A. MacLeod, A simple method for high-rank families of elliptic curves with specified torsion, arXiv:1410.1662.

[13] J.-F. Mestre, Construction d'une courbe elliptique de rang $\geq 12$, C. R. Acad. Sci. Paris Ser. I 295 (1982) 643-644.

[14] The PARI Group, PARI/GP version 2.7.0, Bordeaux, 2014, http://pari.math. u-bordeaux.fr/.

[15] K. Nagao, An example of elliptic curve over $\mathbb{Q}$ with rank $\geq 21$, Proc. Japan Acad. Ser. A Math. Sci. 70 (1994) 104-105.

[16] J. Woo, Arithmetic of elliptic curves and surfaces descent and quadratic sections, PhD thesis, Harvard University, 2010.

A. Dujella

Department of Mathematics

University of Zagreb

10000 Zagreb

Croatia

E-mail: duje@math.hr

J. C. Peral

Departamento de Matemáticas

Universidad del País Vasco

48080 Bilbao

Spain

E-mail: juancarlos.peral@ehu.es

P. Tadić

Department of Mathematics, Statistics and Information Science

Juraj Dobrila University of Pula

52100 Pula

Croatia

E-mail: petra.tadic@unipu.hr

Received: 24.10.2015. 